\title{
EVALUACIÓN DE LA GESTIÓN DE RESIDUOS PELIGROSOS (RESPEL) Y SUS IMPLICACIONES EN EL DESARROLLO SOSTENIBLE DE LAS ACTIVIDADES PRODUCTIVAS EN CINCO MUNICIPIOS DEL DEPARTAMENTO DEL QUINDÍO, COLOMBIA
}

\author{
Andrea Lucía Muñoz-Valencia \\ Carlos Alberto Franco-Cano (iD ${ }^{2}$ \\ Héctor Fabián Triviño-Arbeláez (D) ${ }^{3}$ \\ Ricardo Álvarez-León (D)
}

Recibido el 9 de agosto de 2014, aprobado el 6 de agosto de 2015 y actualizado el 29 de diciembre de 2016

DOI: 10.17151/luaz.2017.44.20

\section{RESUMEN}

Objetivos: Revisar la normatividad de residuos peligrosos en Colombia, su aplicabilidad y reglamentación, en un departamento pequeño que tiene una reducida generación, con el fin de identificar los vacíos existentes y la aplicabilidad de la política ambiental nacional, así como un análisis DOFA en la aplicabilidad de la normatividad de residuos peligrosos en el departamento del Quindío, comprobando la gestión integral y sus implicaciones.Metodología: La revisión incluyó la revisión de la comprensión de la normatividad, así como la bibliografía usada en la formulación de la política, los casos exitosos en otros países y la realización de encuestas en el trabajo de campo a los generadores de residuos, con lo cual se obtuvo la información necesaria para evaluar la aplicación de la Política Nacional en el departamento del Quindío. Resultados: Se visibilizaron las realidades de los generadores de residuos peligrosos en los cinco municipios del Quindío frente a la normativa ambiental y la Política Nacional, se encontró que la normativa nacional excluye a este sector, toda vez que hace exigencias que son difíciles de cumplir, por tanto no se aplica la norma. Discusión: Actualmente, no se promueve a nivel regional y nacional, la gestión de residuos peligrosos ni las opciones que faciliten el cumplimiento de las normas específicas, sin poner en riesgo la sustentabilidad ambiental, la sostenibilidad económica y social de los generadores de residuos peligrosos en los cinco municipios del Quindío. Conclusiones: Como producto de la investigación, se propone un modelo de gestión de residuos peligrosos, con dos variables dependiendo del volumen y características de los residuos generados, que incluye el ajuste normativo, la asociatividad de los generadores, una modificación del marco tarifario (con base en la regulación de los parámetros críticos de recolección y transporte), y el acompañamiento de los sectores comprometidos con la sostenibilidad ambiental, principalmente con la capacitación, formación ambiental y fortalecimiento técnico referido al manejo de los residuos o desechos peligrosos (líquidos, gaseosos, sólidos), orgánicos (que pueden tener procesos de compostaje) e inorgánicos (que pueden ser utilizados como reciclado o reutilizados como logística reversa o inversa) y los inservibles. Existe un gran desafío para un país, en especial cuando éste se enmarca en compromisos de convenios internacionales, al no tener en cuenta la heterogeneidad de las regiones, en cuanto a las capacidades de gestión y eliminación adecuada de residuos.

\section{PALABRAS CLAVE}

Residuos peligrosos, normativa ambiental, gestor, gestión de residuos, política, departamento del Quindío, Colombia. 


\title{
ASSESSMENT OF HAZARDOUS WASTE MANAGEMENT (RESPEL) AND ITS IMPLICATIONS FOR SUSTAINABLE DEVELOPMENT OF PRODUCTIVE ACTIVITIES IN FIVE MUNICIPALITIES OF THE DEPARTMENT OF QUINDIO, COLOMBIA
}

\begin{abstract}
Objectives: To review the regulations for hazardous waste in Colombia, its applicability and regulation, in a small Department that has a reduced generation in order to identify gaps, and the applicability of the national environmental policy, as well as a DAFO analysis in the applicability of the regulations of hazardous waste in the department of Quindío, checking integrated management and its implications. Methodology: The revision included a review of the understanding of the regulations, as well as the literature used in the formulation of the policy, the successful cases in other countries and surveys to waste generators in the field work, with the purpose of obtaining the information needed to evaluate the implementation of the National Policy in the Department of Quindío. Results: The realities of hazardous waste generators became visible in the five municipalities of Quindío against the environmental regulations and the National Policy. It was found that the national legislation excludes this sector since it makes demands that are difficult to fulfill, therefore the rule is not applied. Discussion: Currently, management of hazardous waste or options to facilitate the achievement of specific standards without compromising environmental, economic and social sustainability of the generators of hazardous waste in the five municipalities of Quindío is not promoted at the regional and national level. Conclusions: As a result of the research, a model of hazardous waste management is proposed with two variables depending on the volume and characteristics of waste generated including regulatory setting, the association of the generators, a modification of the rate structure (based on the regulation of critical collection and transport parameters), and the accompaniment of the sectors committed to environmental sustainability, mainly through training, environmental education and technical strengthening relative to the handling of residues or hazardous waste (liquid, gaseous, solids), organic (which may have composting processes) and inorganic (which can be used as recycled or reused as reverse or inverse logistics) and unusable. There is a great challenge for a country, especially when it is part of commitments to international conventions, when it does not take into account the heterogeneity of the regions in terms of management skills and proper waste disposal.
\end{abstract}

\section{KEY WORDS}

Hazardous waste generators, manager, waste management, politics, Department of Quindío, Colombia

\section{INTRODUCCIÓN}

Los esquemas económicos mundiales han cambiado y de un mundo aislado se ha pasado a un mundo interconectado, donde la globalización social, económica y ambiental han sobrepasado los límites de la imaginación, creando una conciencia colectiva mundial, lo que ha permitido desarrollar numerosas convenciones, tratados, cumbres, convenios, en donde los criterios ambientales han sido internalizados como políticas de Estado y de comercio y han causado que países, que antes solo se 
consideraban proveedores de materias primas y en los cuales se ha venido consolidando el desarrollo del aparato industrial, se vean obligados a la instauración de políticas y al establecimiento de una normativa ambiental que les permita insertarse en el nuevo esquema mundial ambiental, como es el caso de Colombia (DNP, 1998).

Como resultado de estos esfuerzos transnacionales para hacerle frente a la creciente problemática generada por estos residuos que presentan alguna característica de peligrosidad, muchas naciones incorporaron o adoptaron en sus legislaciones una serie de normas y de políticas que permitan la protección de los recursos naturales y la salud humana, con las cuales posiblemente se pretende alinearse con los nuevos desafíos mundiales, unificando criterios, procedimiento o protocolos para el control, seguimiento de residuos y a su vez promover el desarrollo sustentable de todas las actividades que generan los residuos con sustancias peligrosas. Un ejemplo de lo anterior, lo enmarcan convenios tan importantes como los de Basilea, Estocolmo, algunas directrices de la Unión de Naciones Andinas, la Unión Europea, entre otras (DNP, 1998).

Lo anterior es claro en la normativa que presentaba el país antes de la Constitución Política de 1991, en donde sobresalían así como la Ley 2 de 1959, el Decreto-Ley 2811 (Código Nacional de Recursos Naturales Renovables y de Protección al Medio Ambiente), reglamentado parcialmente por los Decretos 1715 de 1978, 1741 de 1978, y 02 de 1982, la Ley 9 de 1979, y el Decreto 1594 de 1984, entre otros (ÁlvarezMonsalve, 2009).

Los cinco acuerdos de Río constituyen quizás la respuesta política más universal y articulada para establecer un régimen internacional de cooperación con la plena incorporación de la dimensión ambiental. Eso se ha reafirmado también en la Declaración de Río, que califica el crecimiento económico y social al remitirse a los fundamentos de los procesos de producción y consumo, y al incorporar el concepto de Estocolmo de "responsabilidades comunes aunque diferenciadas", que quedó plenamente reconocido en el principio siete de la citada declaración (PNUMA, 2002).

Conforme a la normativa ambiental colombiana, los residuos o desechos peligrosos (RESPEL), comprenden aquellos residuos que por sus características corrosivas, reactivas, explosivas, inflamables, infecciosas, tóxicas o radiactivas, pueden causar riesgo a la salud humana o al medio ambiente. Asimismo, se considera RESPEL a los envases, recipientes y embalajes que han estado en contacto con ellos (Decreto 4741 del 30 de diciembre de 2005 expedido por el Ministerio de Ambiente, Vivienda y Desarrollo Territorial; presenta los criterios para considerar un residuo como peligroso). Estos se relacionan a continuación: Residuo infeccioso, Residuo combustible, Residuo inflamable, Residuo explosivo, Residuo radioactivo, Residuo volátil, Residuo corrosivo, Residuo reactivo y Residuo tóxico. La generación de estos RESPEL está dada por las actividades productoras y consumidoras de bienes o servicios: el sector manufacturero que transforma materia prima en bienes, el sector agroindustrial que comprende procesos de transformación y producción de plantas y animales in situ, el sector destinado a la prestación de servicios y el sector doméstico (MAVDT, 2005).

En el estudio que adelantó el Departamento Nacional de Planeación, se realizó una estimación de los residuos sólidos y peligrosos generados, tomando como base índices de generación por empleado. Según este estudio, la producción de residuos sólidos industriales a nivel nacional puede alcanzar alrededor de 6.300 ton/día y de estos se estima que cerca de 540 ton/día son RESPEL (Sánchez y Uribe, 1994).

En la presente investigación se hace una revisión de la Política Nacional de Residuos o Desechos Peligrosos, así como de las normas complementarias expedidas en 
relación con el tema, con el fin de revisar su proceso de construcción y encontrar posibles debilidades en la estructuración de la misma y la forma en que esto puede estar afectando a los generadores de pequeñas cantidades de RESPEL. (MuñozValencia et al., 2013).

Igualmente, en el presente trabajo se realizó un análisis del enfoque dado a la Política Nacional y de las obligaciones establecidas para los generadores de RESPEL, encontrando una carga normativa fuerte uniforme, que no distingue entre generadores con volúmenes grandes o pequeños, peligrosidad del residuos, lo que puede generar costos muy altos para los pequeños que pueden poner en riesgo su sostenibilidad financiera. (CRQ et al., 2003).

Al encontrar normas rígidas en materia de gestión integral de RESPEL, se desarrolló y aplicó un instrumento de captura de información, con el fin de poder establecer el grado de cumplimiento de la norma por parte de los generadores y cómo esto podía afectar el desarrollo de sus actividades, su competitividad y su sostenibilidad.

Igualmente, se realizó un análisis de las alternativas de gestión y eliminación a las que pueden acceder los generadores de RESPEL ubicados en el departamento del Quindío, considerando que es un departamento con bajos índices de generación de este tipo de residuos y cómo esto afecta la oferta de las empresas gestoras o eliminadoras.

Para tal fin, se realizó un inventario de las empresas gestoras ubicadas cerca al departamento del Quindío, con el fin de evidenciar las opciones de eliminación y la oferta de residuos que gestionaban, encontrando que la mayoría se encuentran en los departamentos del Valle del Cauca, Cundinamarca y Antioquia, y para las cuales un parámetro crítico para el establecimiento de la tarifa a cobrar lo impone el costo del transporte, por esta razón, el trabajo presenta una propuesta de reordenamiento del esquema de recolección y gestión de RESPEL, con el fin de beneficiar en precios al generador pequeño y garantizar el cumplimiento de la normativa ambiental y la sostenibilidad económica del mismo.

Finalmente, con base en la información encontrada en la bibliografía y en el campo, se pueden determinar algunas debilidades en la normativa ambiental aplicable y se presentan alternativas de solución que buscan que la Política Nacional de residuos sea más incluyente y permita una sostenibilidad económica y cumplimiento de la normativa ambiental para pequeños generadores de residuos, en especial aquellos ubicados en departamentos con baja generación de RESPEL como el departamento del Quindío.

\section{MATERIALES Y MÉTODOS}

\section{Tipo de investigación}

El presente estudio tiene un enfoque de tipo cualitativo y posee un diseño descriptivo a partir de información numérica, pues trata a partir de datos tomados en campo y de información secundaria presentar un diagnóstico a partir del cual se determina una problemática y se presentan posibles alternativas que permitan mejorar la situación de los sujetos objetivo.

\section{Población muestreada}


La población está conformada por generadores de RESPEL en el departamento del Quindío. Se tomó una muestreo probabilístico tipo aleatorio simple a 600 generadores, correspondiente al $51,28 \%$ del total del universo estimado en el diagnóstico realizado por la Corporación Autónoma Regional del Quindío (CRQ) en el 2006, y de la cual se definió la muestra definitiva, mediante la fórmula de número aleatorio, lo que dio como resultado 181 encuestas. Se tomó un 0,5 de probabilidad de ocurrencia de error en la aplicación de la encuesta, un nivel de confianza del 99\% y un error máximo del $8 \%$. (Triviño-Arbeláez, 2006)

La selección de los encuestados se realizó de manera aleatoria, en las áreas en donde se presentaban pequeños o medianos establecimientos generadores de RESPEL.

\section{Instrumentos}

La encuesta aplicada a los generadores de RESPEL fue diseñada con el fin de capturar la información básica del establecimiento generador y a través de 13 preguntas tipo abierto y cerradas, relacionadas con la generación, gestión interna y externa de residuos y los posibles inconvenientes presentados para el cumplimiento de la normativa ambiental aplicable a la materia.

La encuesta fue diseñada con el apoyo de la CRQ, y aplicada por técnicos de la misma entidad quienes apoyaron la ejecución del presente trabajo investigativo.

Para la aplicación de las encuestas en campo se consideró, entre otros aspectos, que no incluyera a los generadores de residuos hospitalarios y similares, porque este tipo de residuos posee otra normativa que se escapa del alcance de la presente investigación, centrándose en establecimientos que desarrollan actividades industriales, comerciales, de servicios, institucionales, agroindustriales, identificados por la CRQ (Triviño-Arbeláez, 2006).

Para garantizar una adecuada aplicación del instrumento de captura de información se realizaban reuniones periódicas con los técnicos de apoyo, en las que se verificaba el trabajo realizado y se realizaba la validación y consolidación de encuestas, y en caso de encontrar infracciones ambientales se procedía a coordinar las acciones de control por parte de la Autoridad Ambiental.
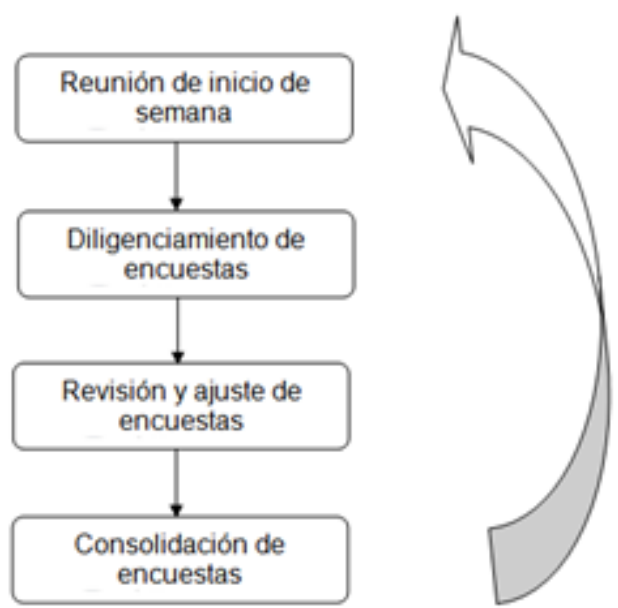

Figura 1. Fase de aprestamiento. 
Las encuestas se sistematizaron en una hoja de cálculo con el fin de incorporar toda la información que hace parte del resultado final del diagnóstico y sobre el cual se plantearan las alternativas de solución.

\section{Procedimiento}

Antes de la aplicación del instrumento de captura de información, se realizó una revisión bibliográfica sobre la Política Nacional de Residuos o Desechos Peligrosos, haciendo énfasis en su diseño y alcance, al igual que la normativa derivada de esta política y la cual se aplica a todos los generadores de RESPEL.

Se revisaron trabajos anteriores realizados en el departamento del Quindío, en especial aquellos tendientes a la identificación de los generadores de RESPEL, con el fin de establecer su ubicación, tipo de generador, potencial de generación, tipo y cantidad de residuo peligroso generado.

Para la aplicación de las encuestas inicialmente se diseñó y elaboró el cuadernillo, para lo cual se tuvieron en cuenta instrumentos anteriores aplicados en el Quindío en materia de RESPEL que permitieran adquirir la información requerida para los fines de la investigación; se realizaron pruebas en campo, se validaban, se corregía su contenido, hasta lograr un instrumento acorde con las necesidades, de fácil diligenciamiento, interpretación y entendimiento por parte del encuestado.

Durante seis meses se aplicaron 600 encuestas a los potenciales generadores de RESPEL en las áreas urbanas y suburbanas de los cinco municipios seleccionados. La información primaria capturada corresponde a promedios mensuales de generación en dichos establecimientos.

Las encuestas fueron aplicadas en los municipios de Armenia, Calarcá, Circasia, La Tebaida y Montenegro, porque estos municipios son los que tienen mayor cantidad de generadores de RESPEL de conformidad con el precitado diagnóstico hecho por la CRQ (Triviño-Arbeláez, 2006).

A continuación se presenta, en la Figura 2, el esquema empleado para el procesamiento de las encuestas.

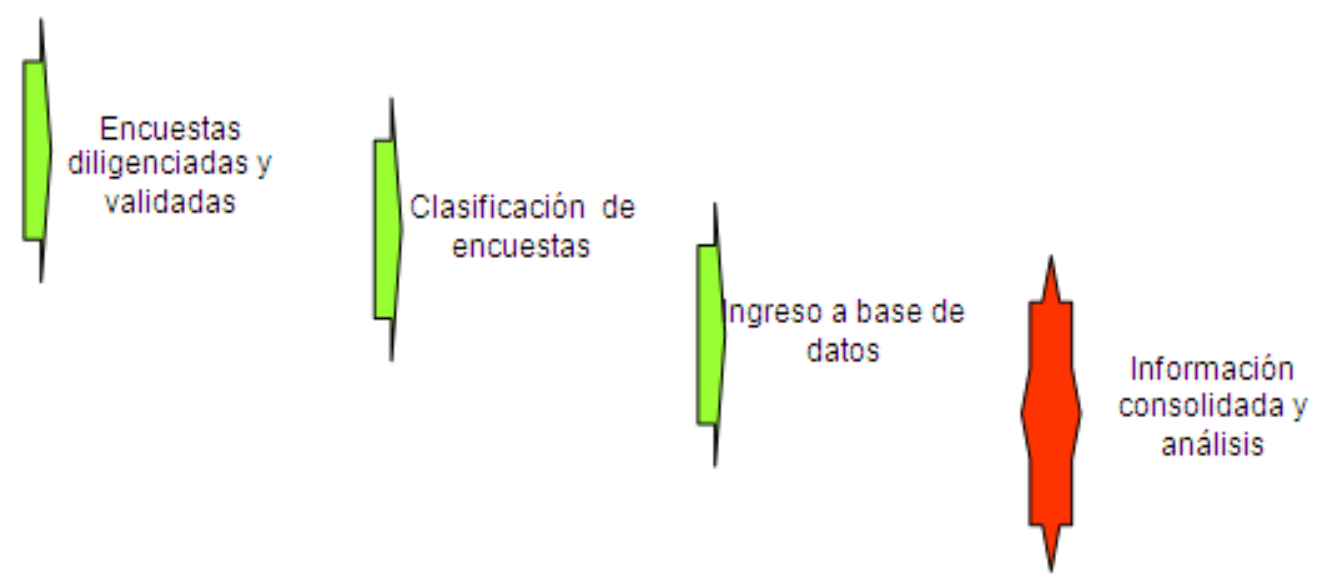

Figura 2. Secuencia utilizada para procesar las encuestas realizadas.

Seguido al procesamiento de la información se realizó la categorización de variables y análisis de la misma, conforme a las obligaciones establecidas en la normativa ambiental, para finalmente correlacionar las obligaciones impuestas por esta normativa 
y la Política Nacional a los pequeños y medianos generadores del Quindío, analizarla a la luz de los conceptos de desarrollo sostenible y en especial en sustentabilidad social, económica y ambiental de la actividad productiva.

Igualmente, se revisaron algunos modelos aplicables a la Política Nacional de Residuos o Desechos Peligrosos, alternativas de normativa ambiental aplicada en países de la región, Estados Unidos, y la Comunidad Económica Europea con el fin de tener un mayor panorama de las situaciones en esos países y construir una base conceptual sobre posibles alternativas de gestión basadas en esas experiencias.

\section{RESULTADOS}

Los establecimientos visitados se agruparon por código internacional industrial uniforme -CIIU-, con el fin de estandarizar las actividades que fueron objeto de estudio y facilitar su clasificación. A continuación se presentan, en la Figura 3, los resultados de las actividades encontradas en el trabajo de campo:

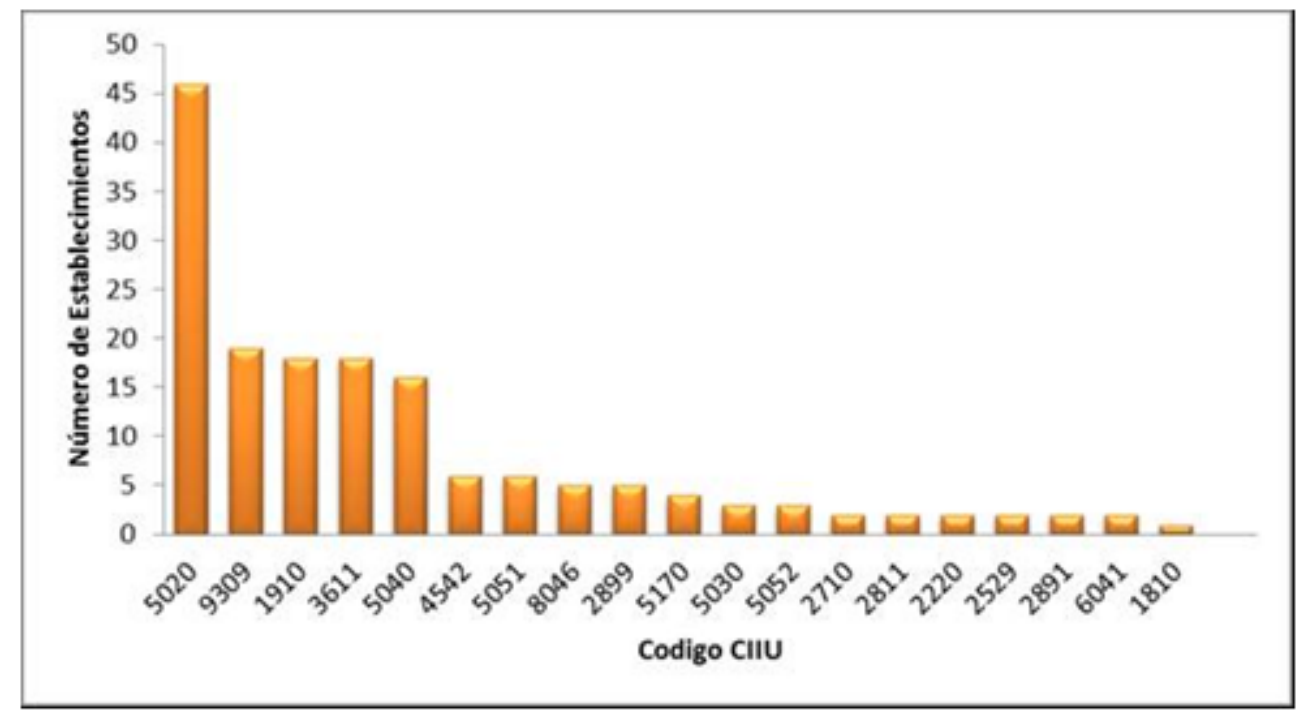

Figura 3. Códigos de las actividades CIIU.

En relación con el tamaño de las empresas encontradas en el campo, se encontró que en el Quindío predominan las pequeñas empresas, en las cuales el número de empleados se encuentra por debajo de 8, siendo consistente con la hipótesis planteada para la presente investigación en relación con el tamaño de las actividades generadores en el Quindío, lo que puede llevar a inferir que la generación por establecimiento es igualmente pequeña y poco atractiva para los gestores de RESPEL. En la Figura 4 se presenta la distribución porcentual del tamaño de los establecimientos o empresas visitadas en el Quindío. 


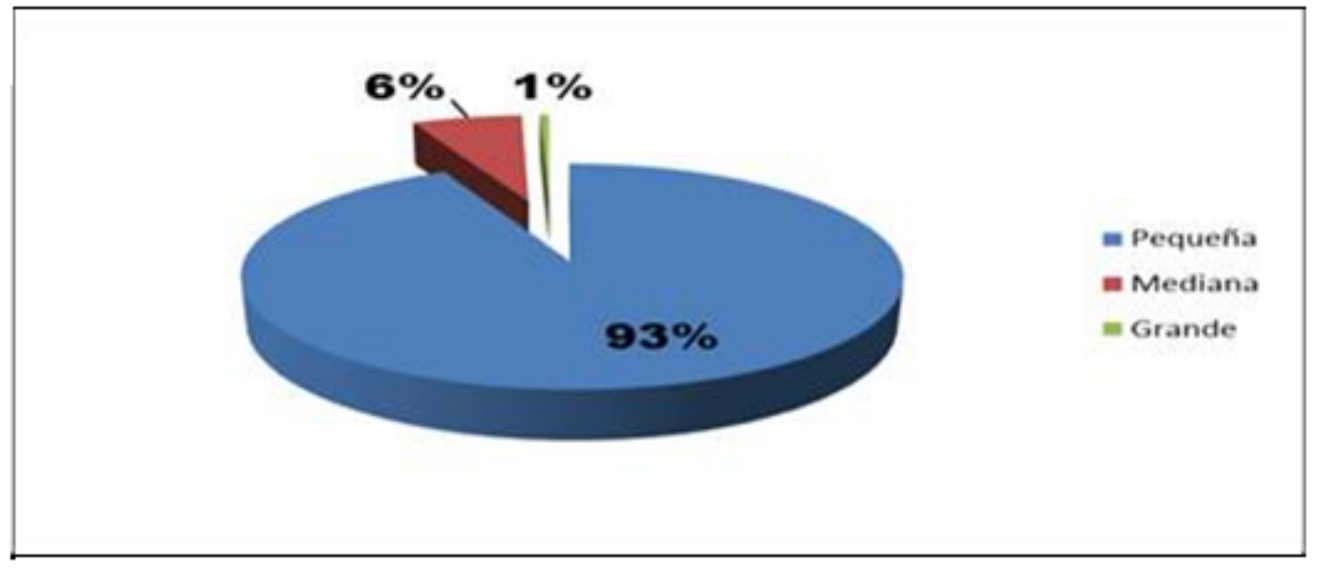

Figura 4. Tamaño de las empresas visitadas.

Se encontró en las visitas realizadas, que entre los RESPEL de mayor generación en el Quindío se encuentran los aceites, envases de aceites, pinturas y disolventes, que son generados principalmente en las actividades de mecánica vehicular y no vehicular, así como en la fabricación de muebles y en establecimientos dedicados al terminado de superficies, pulimientos, pinturas, entre otros, como se muestra en la Figura 5.

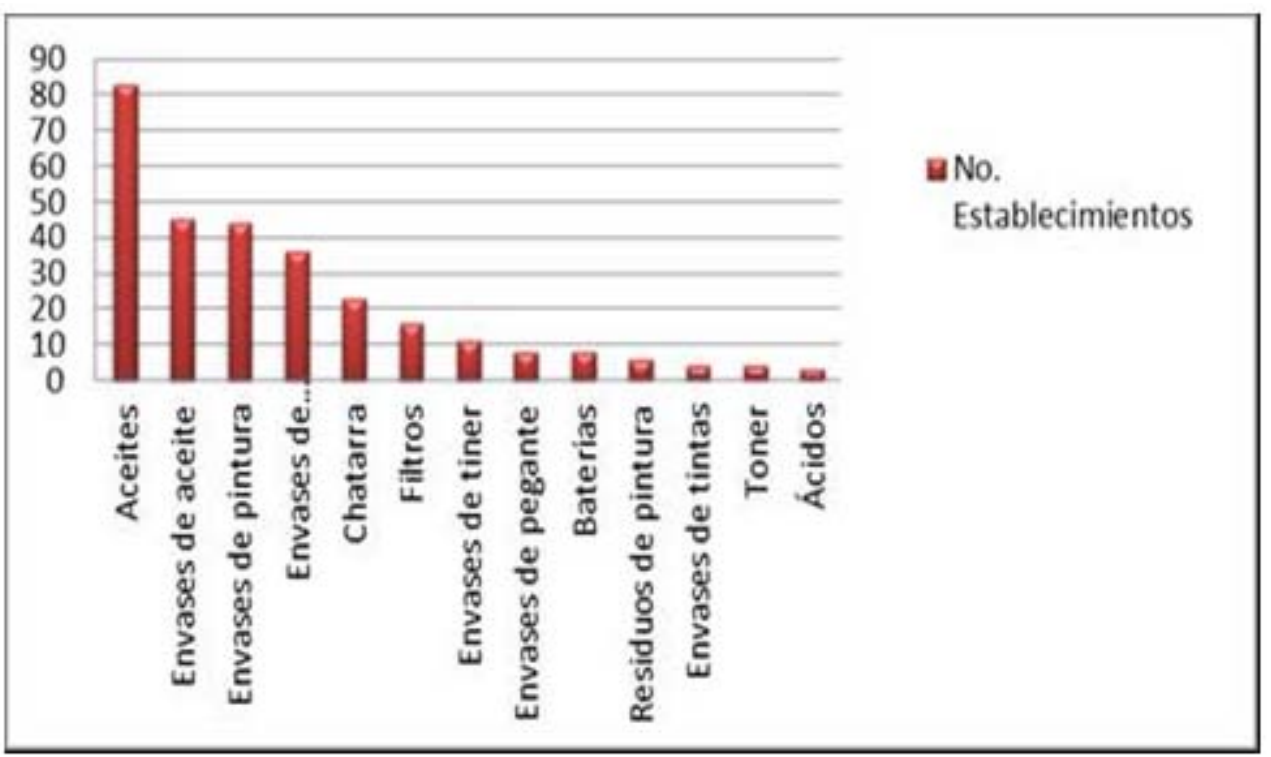

Figura 5. Residuos generados en las empresas seleccionadas.

Durante el análisis de los resultados se evaluaron las diferentes alternativas existentes en el Quindío para el manejo, gestión, tratamiento o disposición final que se le viene dando a cada uno de ellos por parte de los generadores, encontrando, en su mayoría, que estos disponen sus residuos de manera inadecuada, tales como chatarrerías, venta a terceros posiblemente sin permisos o autorizaciones ambientales para la realización de tal labor, además aproximadamente el $22 \%$ de los encuestados no respondieron la pregunta correspondiente.

Lo anterior, puede deberse a que el $75 \%$ de los encuestados adujeron no conocer la norma relacionada con gestión integral de RESPEL, lo que pudo haber dificultado el cumplimiento de la misma por parte de los generadores. A continuación se muestra, en 
laFigura 6, el grado de conocimiento de la norma por parte de los generadores visitados.

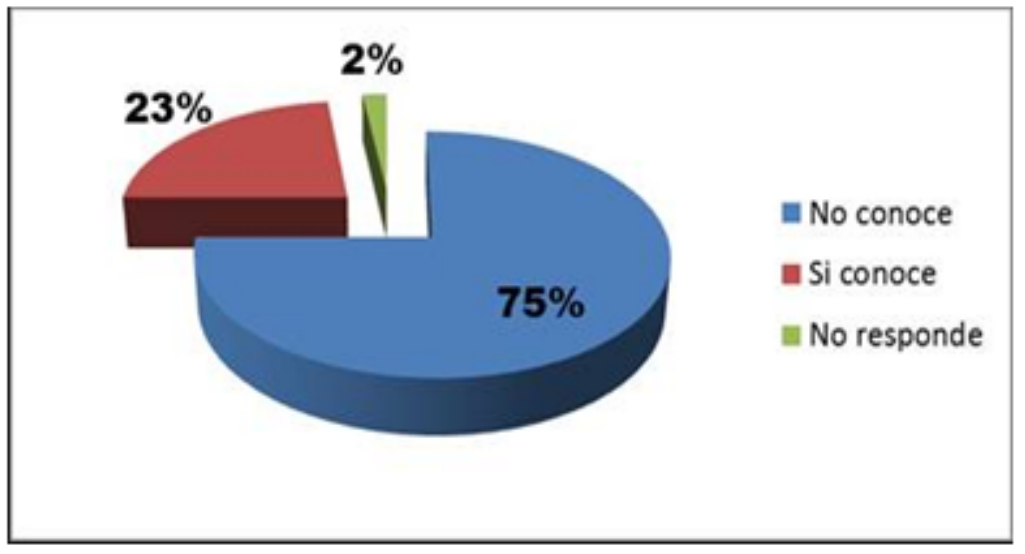

Figura 6. Conocimiento de la norma de RESPEL.

Al consultar a los generadores sobre la dificultad en la aplicación de la norma de RESPEL, en el tema de eliminación, tratamiento de disposición final, estos manifestaron que era difícil toda vez que en el Quindío no existía una oferta de empresas y las existentes tienen costos altos, lo que puede perjudicar la estabilidad de las empresas, las cuales llevan los residuos recolectados en el Quindío a instalaciones ubicadas por fuera del mismo (Figura 7).

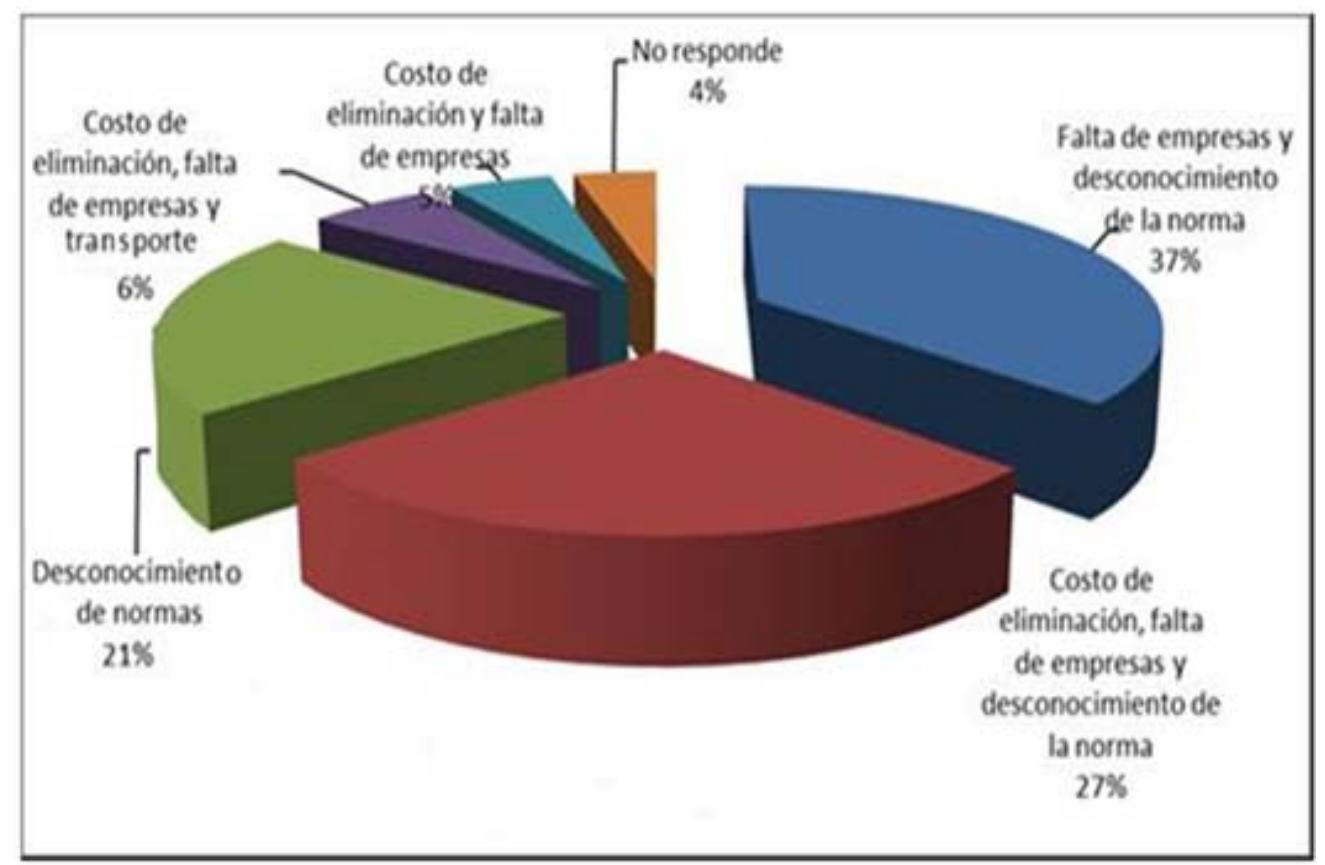

Figura 7. Obstáculos para la aplicación de la norma.

Con el fin de corroborar lo anterior se realizó un inventario de las empresas gestoras o eliminadoras de RESPEL, en especial las que estaban en capacidad de tratar o eliminar los RESPEL generadores en el Quindío, encontrando que efectivamente todas están por fuera y a distancias cuyo factor podría afectar la tarifa final aplicada al 
generador (Figura 7), más aun cuando éste solo entrega pequeños volúmenes, si se le compara con otros departamentos (Figura 8).

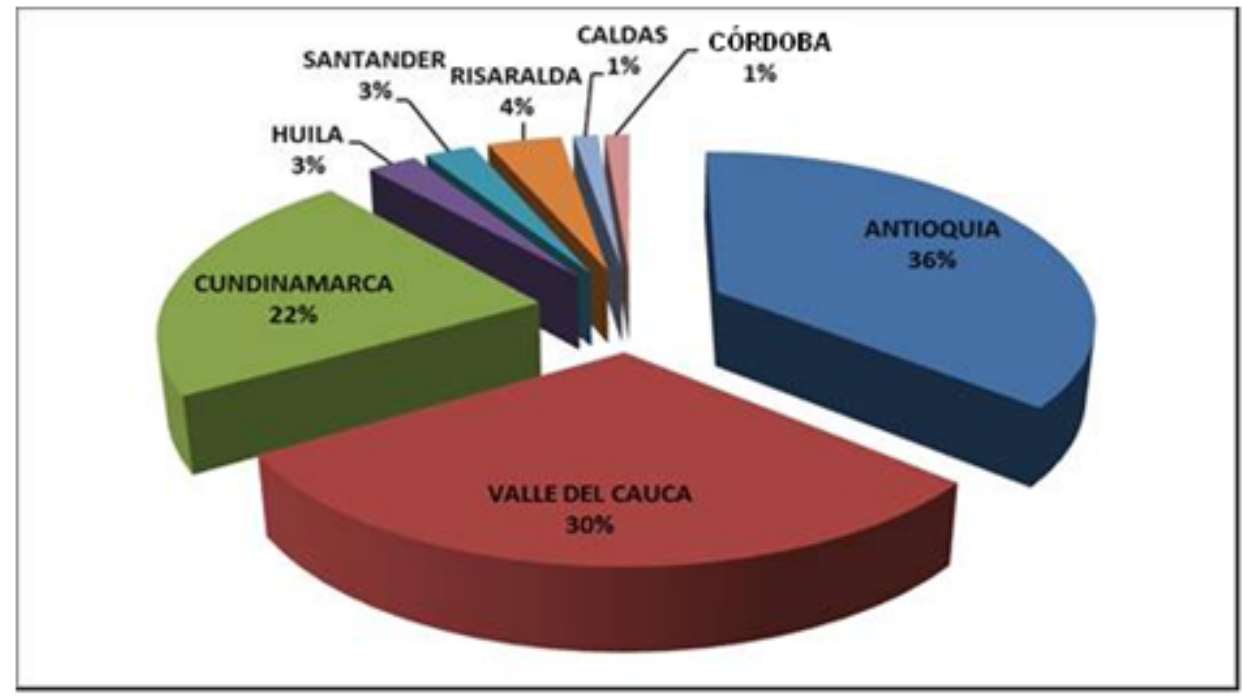

Figura 8. Distribución de las empresas gestoras de RESPEL en Colombia.

\section{CONCLUSIONES}

La norma para el manejo de RESPEL como está concebida es para todos los generadores, pero no tiene en cuenta muchas de las necesidades de los pequeños generadores, pues no establece opciones para las regiones sin servicio de gestores, lo cual constituye una debilidad de la misma e impacta negativamente su aplicación, en las regiones que no presentan autosuficiencia en el tratamiento, aprovechamiento, eliminación o disposición final de sus RESPEL. Como quedó demostrado en la presente investigación, esta situación impacta especialmente a los pequeños generadores de residuos, porque les impone obligaciones difíciles de cumplir, por no contar con las alternativas exigidas en el área y deben incurrir en gastos elevados para el pago del transporte, el tratamiento y disposición final, con empresas de otros departamentos.

Se realiza inadecuada gestión por parte de los generadores, debido a la falta de conocimiento, o interés de los mismos en temas de residuos. Una estrategia de capacitación, actualización y educación ambiental en el tema del manejo integral de RESPEL puede reducir el riesgo de los pequeños generadores a disponer inadecuadamente sus residuos y permite una gestión técnicamente adecuada, ambientalmente sustentable y económicamente viable.

Las evidencias encontradas en la presente investigación, conllevan a determinar que la Política Nacional para la gestión integral de RESPEL así como las normas emitidas para su aplicación, presentan vacíos en su validez para regiones o departamentos no industrializados, como el caso del departamento del Quindío, dado que esta impone obligaciones a los generadores, especialmente en el componente de tratamiento, aprovechamiento, eliminación o disposición final de sus RESPEL, pero no se promueve el establecimiento de alternativas de gestión locales adecuadas para los 
mismos. Esto conlleva a buscar alternativas de manejo en otras regiones generando costos excesivos, lo que afecta la estabilidad de los pequeños establecimientos o microgeneradores de RESPEL.

Las normas ambientales relacionadas con el tema de RESPEL no son aplicables a todo el territorio nacional, debido a la heterogeneidad del mismo y las características geográficas, sociales y económicas de las regiones, por lo que estas deben presentar un mayor ajuste local, es decir, se debe buscar un mecanismo que permita su flexibilidad en las regiones tal y como se presenta en otros países de la región, buscando una mejor aplicabilidad a las realidades regionales.

Los modelos propuestos para promover el cumplimiento de la normativa y la Política Nacional de Residuos o Desechos Peligrosos, están basados en el fortalecimiento de los sistemas de recolección a pequeños generadores, dado que como quedó demostrado en el presente trabajo, se estima que este sector en el departamento del Quindío lo comprende alrededor del 95\% de los generadores de RESPEL. Para mejorar la gestión se tomaron modelos exitosos en otras regiones del país, basados en estrategias de acopio y creación de organizaciones para la recolección de RESPEL, con el acompañamiento permanente de las empresas eliminadoras que cuenten con las licencias ambientales.

Se identifica una preocupación de los generadores relacionada con la sostenibilidad de sus empresas bajo los parámetros normativos de RESPEL, ya que para dar cumplimiento de los mismos deben incurrir en gastos económicos elevados, para disponer los RESPEL generados, debido a que las empresas que prestan el servicio provienen de zonas alejadas del área de generación, lo que incrementa los costos a pagar por el rubro de transporte.

Existe una serie de obligaciones establecidas por la norma ambiental vigente aplicables a generadores de RESPEL, tal como el diseño e implementación de Plan de Gestión de Residuos o Desechos Peligrosos en sus establecimientos, situación que representa costos adicionales, los cuales se vuelven sensibles para los microgeneradores. Al revisar las normas internacionales tales como las de la EPA de Estados Unidos de América (EPA, s.f., 1980, 2003) e ICT de Chile, se observa que esta obligación solamente la tienen aquellos establecimientos que generan más de cierta cantidad mensual de RESPEL o que las características de peligrosidad de los mismos ameriten un manejo especial, eliminando esta obligación para algunos generadores pequeños.

Los planes de devolución posconsumo tal y como están estructurados, no contribuyen a la gestión adecuada de los RESPEL que pretenden recolectar, toda vez que tal y como lo estable la norma, los fabricantes e importadores de los productos que generan RESPEL después de su uso, solamente deben presentar ante el Ministerio de Ambiente y Desarrollo Sostenible el cumplimiento de una meta global de recolección nacional de estos residuos, por lo tanto, las campañas de recolección de productos posconsumo solamente se centran en ciudades principales $y$ en algunos departamentos, y se descuida otras regiones, las cuales por sus niveles de consumo y generación de residuos no son significativas para el gestor.

La Política Nacional de Residuos o Desechos Peligrosos promulgada por Colombia en 2005, a pesar de presentar una estructura adecuada para una política de gestión, no involucró en su estructuración y definición las necesidades de las regiones y departamentos con bajos volúmenes de generación y no presentaba alternativas de ajuste para las regiones. 
Es importante que los entes territoriales participen activamente en la identificación, registro y control de los generadores de RESPEL, en especial en los microgeneradores, pequeños y medianos, toda vez que es en estos segmentos en donde es más difícil el control por las autoridades ambientales, debido a la dificultad para su identificación y detección. Por ende, al estar identificados por estos, facilita el establecimiento de alternativas colectivas de gestión y saneamiento a través de empresas que presten el servicio especializado de recolección, tratamiento y disposición final de RESPEL, mediante las diferentes opciones enunciadas en esta contribución, tales como: creación de empresas asociativas entre generadores para gestionar sus residuos, establecimiento de áreas de servicio exclusivo, acompañados de la flexibilización normativa especialmente la ambiental y en lo referido a licenciamiento ambiental según consideraciones técnicas y las características de peligrosidad del residuo o desecho gestionado.

Se deben considerar seriamente casos exitosos de otros países, tales como Estados Unidos de América, la Unión Europea, Argentina, Chile, entre otros, en los cuales las políticas y normas ambientales relacionadas con RESPEL están sujetas a ajustes locales y regionales, lo que facilitó la implementación de las mismas debido al acercamiento a las realidades de cada región, pasando de ser unas normas excluyentes a un modelo incluyente que respeta las características regionales, culturales y económicas de cada localidad.

Estados Unidos, por ejemplo, cuenta con una infraestructura adecuada para el tratamiento y disposición de RESPEL (Martínez et al., 2005). Cada Estado adecúa las Normas Federales DE LA EPA, a las realidades y condiciones estatales (EPA, 2003), lo que le permite garantizar una aplicación adecuada de la normativa en su territorio, aunado a la responsabilidad ambiental que presentan los generadores, gestores y eliminadores de RESPEL.

\section{REFERENCIAS}

- Álvarez-Monsalve, C.A. (2009). Gestión integral de residuos sólidos: Minimización, almacenamiento, manipulación, transporte, aprovechamiento, tratamiento $y$ eliminación. Disposición Final. Manizales, Colombia: Universidad de Manizales.

- CRQ. (2009). Plan de Gestión Integral de Residuos o Desechos Peligrosos para el Departamento del Quindío. Corporación Autónoma Regional del Quindío. Armenia (Quindío), Colombia.

- CRQ / CVC / CARDER / CORTOLIMA. (2003). Eco Región del Eje Cafetero. Armenia (Quindío), Colombia. Corporación Autónoma Regional del Quindío / Corporación Autónoma Regional del Valle del Cauca / Corporación Autónoma Regional del Risaralda / Corporación Autónoma Regional del Tolima.

- DNP. (1998). Comercio, Medio Ambiente y Desarrollo. Dirección Nacional de Planeación). Bogotá D.C., Colombia. 
- EPA (1980). Hazardous waste management system, Part III, Identification and listing of hazardous waste. Enviromental Protection Agency. Washington D.C. (USA). Federal Register, May 19, 45(98).

- EPA. (2003). Manejando sus residuos peligrosos. Una guía para empresas pequeñas. Enviromental Protection Agency. Washington D.C. (USA).

- $\quad$ EPA. (s.f.). Managing non-hazardous solid waste. Chapter II. Washington D.C. (USA de Ambiente, Vivienda y Desarrollo Territorial).). pp. 1-8. Disponible en: http://www.epa.gov/rcc

- Martínez, J.; Mallo, M.; Lucas, R.; Álvarez, J.; Salvarey, A. y Gristo, P. (2005). Guía para la Gestión Integral de Residuos Peligrosos - I, Fundamentos. Montevideo (Uruguay). Centro Coordinador del Convenio de Basilea para América Latina y el Caribe.

- MAVDT (2005). Decreto4741 del 30 de diciembre de 2005. Ministerio de Ambiente, Vivienda y Desarrollo Territorial. Bogotá D.C. (Colombia).

- Muñoz-Valencia, A.L.; Triviño-Arbeláez, H.F. y Franco-Cano, C.A. (2013). Análisis de la aplicabilidad de la política ambiental nacional para la gestión integral de residuos o desechos peligrosos en el departamento del Quindío y sus implicaciones en el desarrollo sostenible de las actividades productivas en el territorio. Tesis de Magíster. Facultad de Ciencias Contables, Económicas y Administrativas. Maestría en Desarrollo Sostenible y Medio Ambiente. Universidad de Manizales, Manizales (Caldas) Colombia.

- PNUMA. (2002). La transición hacia el desarrollo sustentable. Perspectivas para América Latina y el Caribe. México D.F., México: Programa de las Naciones Unidas para el Medio Ambiente.

- Sánchez, E. y Uribe, E. (1994). Contaminación industrial en Colombia. Bogotá D.C., Colombia: DNP/PNUD.

- Triviño-Arbeláez, H.F. (2006). Diagnóstico y problemática ambiental de la generación de residuos peligrosos en el Departamento del Quindío. Armenia (Quindío), Colombia: Corporación Autónoma Regional del Quindío. 
1. $\mathrm{CRQ}$. Magister en Desarrollo Sostenible y Medio Ambiente.andreamvalencia@yahoo.com. ORCID: 0000-0002-3714-0440

2. ASOCARS. Magister en Desarrollo Sostenible y Medio Ambiente.francocano@gmail.com. ORCID: 0000-0002-2143-7477

3. CRQ. Magister en Desarrollo Sostenible y Medio Ambiente.hectorfabiantrivino@gmail.com. ORCID: 0000-0003-4860-0606

4. Universidad de Manizales. Magister en Oceanografía Biológica y Pesquera.ricardoalvarezleon@gmail.com. ORCID: 0000-0002-9668-099X

Para citar este artículo: Muñoz-Valencia, A.L.; Franco-Cano, C.A.; Triviño-Arbeláez, H.F. y Álvarez-León, R. (2017). Evaluación de la gestión de residuos peligrosos (RESPEL) y sus implicaciones en el desarrollo sostenible de las actividades productivas en cinco municipios del departamento del Quindío, Colombia. Revista Luna Azul, 44, 334-347. DOI: 10.17151/luaz.2017.44.20. Recuperado de http://200.21.104.25/lunazul/index.php/component/content/article?id=241

Esta obra está bajo una Licencia de Creative Commons Reconocimiento CC BY

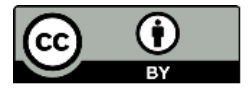

